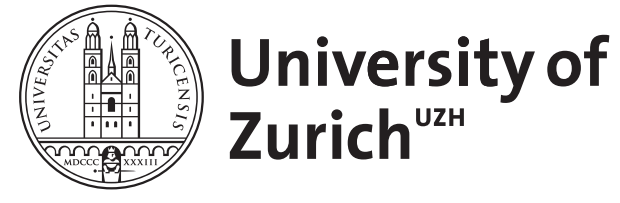
Archive

University of Zurich

University Library

Strickhofstrasse 39

CH-8057 Zurich

www.zora.uzh.ch

Year: 2012

\title{
Zweitmeinung in der Beurteilung der Arbeitsfähigkeit
}

Thöne, H J

DOI: https://doi.org/10.1024/1661-8157/a001103

Posted at the Zurich Open Repository and Archive, University of Zurich

ZORA URL: https://doi.org/10.5167/uzh-67487

Journal Article

Accepted Version

Originally published at:

Thöne, H J (2012). Zweitmeinung in der Beurteilung der Arbeitsfähigkeit. Praxis, 101(20):1287-1294.

DOI: https://doi.org/10.1024/1661-8157/a001103 


\section{Zweitmeinung in der Beurteilung der Arbeitsfähigkeit}

Für den grössten Teil unserer Gesellschaft geniesst die berufliche Tätigkeit einen sehr hohen, ja sogar zentralen Stellenwert. Obwohl in Zusammenhang mit dem Beruf auch Begriffe wie Last und Mühsal zu nennen sind, erfüllt er uns in der Regel mit Stolz und Befriedigung. Vor allem ist er Grundlage unserer wirtschaftlichen Existenz. Freiwillige oder erzwungene Umstände können dazu führen, dass jemand seinen Lebensunterhalt nicht selbst verdient und keiner Erwerbstätigkeit nachgeht. Eine Person kann von sich aus entscheiden, temporär oder dauerhaft keine berufliche Tätigkeit auszuüben. Andererseits können Krankheiten oder Unfälle die Arbeitsfähigkeit beeinträchtigen oder aufheben. In solchen Situationen haben Ärztinnen und Ärzte Arbeitsunfähigkeitszeugnisse auszustellen und mittels dieser Krankschreibungen vorzunehmen. Ist die Wiederaufnahme der Erwerbstätigkeit zeitlich nicht absehbar, werden oft ärztliche Zweitmeinungen zur Beurteilung der Arbeitsfähigkeit eingeholt.

Bedeutung von Arbeit und Nicht-Arbeit ((Ü1))

Arbeiten war in der Vergangenheit weit verbreitet mit den Begriffen Ärger, Mühsal oder gar Qual assoziiert. Dies zeigt auch eine Analyse verschiedener europäischer Sprachen. Das französische Wort «travailler» ist aus dem lateinischen «tripotium» entstanden, offenbar einem damals gefürchteten Folterinstrument. Die gesundheitlichen Auswirkungen einer beruflichen Tätigkeit waren negativ behaftet und wurden als potenziell schädlich für Arbeitnehmer angesehen. Andererseits erkannten bereits Sigmund Freud und José Ortega y Gasset positive Effekte einer Arbeitstätigkeit und Albert Camus zog die knappe Bilanz: «Ohne Arbeit ist das Leben scheusslich». Die meisten Abwesenheiten vom Erwerbsleben sind durch vorübergehende Krankheiten oder leichte Unfälle bedingt und dauern wenige Tage. Gemäss Richtlinien der Canadian Medical Association sollten Arbeitnehmer bei Arbeitsausfällen von bis zu fünf Tagen keine Arbeitsunfähigkeitszeugnisse beibringen müssen. Der Patient sollte sich jedoch wegen seiner Gesundheitsstörung in ärztliche Behandlung begeben haben, respektive der Arzt sollte genügend informiert sein über dessen gesundheitliche Probleme. Die Übernahme dieser Richtlinien für die Schweiz hätte zur Folge, dass weniger Arbeitsunfähigkeitszeugnisse ausgestellt werden müssten.

Langdauernde Abwesenheiten vom Arbeitsplatz sind in der Regel durch schwere Krankheiten oder Unfälle bedingt. Die funktionellen Einschränkungen der Betroffenen sind offensichtlich und in den entwickelten Ländern gibt es Netzwerke von sozialen Institutionen, die die finanziellen Folgen der Arbeitsunfähigkeit mildern.

Teilweise entstehen langdauernde Abwesenheiten vom Erwerbsleben auch durch Ereignisse, nach denen ein Grossteil der Arbeitnehmer die berufliche Tätigkeit nur kurzzeitig unterbricht und sie anschliessend wieder aufnimmt. Die vormalige Leistungsfähigkeit wird uneingeschränkt erreicht, allenfalls nach einer Phase der Teilarbeitsfähigkeit.

Seit einigen Jahren unterstützen Ärztekammern in Nordamerika und Grossbritannien die Stossrichtung einer frühzeitigen beruflichen Reintegration. Sie warnen davor, dass eine dauernde Arbeitsunfähigkeitsschreibung denselben Effekt hat, wie ein Medikament, das als Nebenwirkung die mentale, physische und soziale Integrität einer Person zerstört. Eine lesenswerte Studie von Dunstan aus Australien beschreibt im Jahre 2009 eine 
erhebliche Wahrscheinlichkeit, durch eine langdauernde Erwerbslosigkeit in eine frustrane Lebenssituation zu geraten. Beschrieben werden, nebst einem Verfall des psychischen und physischen Wohlbefindens, zwischenmenschliche Probleme, ein Identitätsverlust und eine Selbstwertproblematik, finanzielle Probleme vor allem durch «Frustkäufe», eine generell schlechtere Lebensqualität und eine Zunahme der Suizidalität. Dieselbe Studie erkennt sowohl auf Seiten der Ärzteschaft, wie auch der Patienten nicht immer nur medizinische Gründe für das Bescheinigen einer langdauernden Arbeitsunfähigkeit. Für Ärzte sind das Alter der Betroffenen, die Einschätzung der Arbeitsmotivation und bestehende familiäre Umstände wichtige Mitgründe hierfür. Patienten haben oftmals das Bedürfnis, ein belastendes Umfeld temporär verlassen zu können.

Bereits 1995 zeigte eine Review aus Canada, dass eine berufliche Untätigkeit eine starke Assoziation zum Auftreten von gesundheitlichen Störungen hat. Arbeiten aus Skandinavien, Griechenland und Nordamerika beschreiben den negativen Effekt einer Arbeitslosigkeit auf die Mortalität.

Eine Review von Waddell und Burton in Bezug auf Arbeit und Gesundheitsprobleme aus dem Jahre 2007 hält fest, dass der Nutzen einer Berufstätigkeit für die Gesundheit die damit verbundenen Gefährdungen aufwiegt und somit grösser ist, als die Gefahren einer Gesundheitsschädigung durch eine dauernde Arbeitsunfähigkeit. Die Autoren kontrastieren diese Feststellung zu der immer grösser werdenden Zahl an langdauernd arbeitsunfähig Geschriebenen, der wachsenden Anzahl Erwerbsunfähiger und der Zunahme der Frühpensionierten aus gesundheitlichen Gründen. Sie kommen zum Schluss, dass es in diesem Punkt ein gesellschaftliches Umdenken braucht.

Generell sind Krankschreibungen für Mediziner keine einfache Tätigkeit. Bei der Einschätzung der Dauer und des Ausmasses einer Arbeitsunfähigkeit sind die betreuenden Ärzte meist auf sich alleine gestellt. Es kommt vor, dass sie in einen Konflikt geraten zwischen ihren Rollen als Behandelnde und Fürsorgende ihrer Patienten einerseits und neutralen Experten, die auch rechtliche Vorgaben sowie die Interessen der Allgemeinheit mit einzubeziehen haben, andererseits. Zudem bestehen ärztlicherseits häufig keine Kenntnisse bezüglich des genauen Tätigkeitsprofils, das Patienten im beruflichen Alltag zu erfüllen haben. Hinderlich sind subjektive Krankheitskonzepte einzelner Patienten, die teilweise von der Lehrmeinung erheblich abweichen können. Zu guter Letzt wird das Thema einer Arbeitsunfähigkeit oftmals am Schluss einer Konsultation kurz vor oder auch nach der Verabschiedung thematisiert, so dass ein erheblicher Zeitdruck besteht und wenig Möglichkeit vorhanden sind, sich gedanklich mit der Thematik auseinanderzusetzen.

Untersuchungen aus den USA aus dem Jahre 2000 zeigen, dass Ärzte durch Krankschreibung einen Beitrag für eine unnötig lange Arbeitsunfähigkeit leisten. Hierfür gibt es verschiedene Gründe. Sie haben manchmal Mühe, einen Patienten, der sich eine Rückkehr ins Erwerbsleben nicht zutraut, diesbezüglich zu motivieren, oder sie haben schlicht zu wenige Angaben zu seiner beruflichen Tätigkeit. Sie haben Schwierigkeiten, Tätigkeiten zu definieren, die für einen Patienten ungünstig sind oder dieser nicht ausführen kann. Arbeitnehmer lehnen es manchmal ab, eine angepasste Arbeit anzunehmen. Arbeitgeber sind möglicherweise nicht willens oder in der Lage, temporär oder dauerhaft geeignete Arbeitsplätze zur Verfügung zu stellen. Ärzte stehen zwischen manchmal diametral auseinandergehenden Interessen von Arbeitnehmern und Arbeitgebern. Teilweise sind Patienten bei zwei Ärzten in Behandlung und deren Auffassungen in Bezug auf eine Arbeitsunfähigkeit sind unterschiedlich.

Ist ein Arbeitnehmer aus gesundheitlichen Gründen nicht in der Lage, in seinen angestammten Tätigkeitsbereich zurückzukehren, verlangen Arbeitgeber, Taggeldversicherer, Invaliden- und Unfallversicherer Informationen von den behandelnden Ärzten oder sie holen sich Zweitmeinungen von unabhängigen Medizinern ein. Mithilfe der Zweitmeinungen soll erreicht werden, dass möglichst zeitnah Massnahmen zur beruflichen Reintegration von Menschen, die aus gesundheitlichen Gründen langzeiterwerbslos sind, getroffen werden können. Weiter ist das Einholen einer medizinischen Zweitmeinung hilfreich, damit zustehende Versicherungsleistungen problemlos geleistet werden und man versicherungsmedizinische Fallstricke vermeidet. 
Praktisches Vorgehen ((Ü1))

Es sei betont, dass eine Zweitmeinung zur Beurteilung der Arbeitsfähigkeit ein medizinischer Bericht und kein gerichtsverwertbares Gutachten ist. $\mathrm{Zu}$ dessen Erarbeitung ist keine vertiefte Auseinandersetzung mit Vorbeurteilungen notwendig. Der Inhalt fokussiert sich auf die Fragestellung.

Obwohl eine Zweitmeinung unvoreingenommen geäussert wird, ist es für die jeweiligen Ärztinnen und Ärzte hilfreich, wenn eine möglichst detaillierte Beschreibung, der von einer Person zu erledigenden Verrichtungen vorliegt und, wenn möglich, eine Beschreibung der Arbeitsumgebung (z.B. Hitze, Kälte, Staub, Feuchtigkeit, Tätigkeiten im Freien, etc.). Auch die Überlassung medizinischer Unterlagen durch den Auftraggeber zur Einsicht macht Sinn, damit der Verlauf einer prolongierten Arbeitsunfähigkeit rekonstruiert und nachvollzogen werden kann.

Es lohnt sich, mit einem Patienten das sogenannte «Haus der Arbeitsfähigkeit», wie es im Finnish Institute of Occupational Health im Jahre 2004 entwickelt wurde, zu bauen. Dieses besteht aus vier Geschossen, die sich alle unter dem Dach der Arbeitsfähigkeit befinden. Im Erdgeschoss, quasi das Fundament bildend, wohnt die Gesundheit. Aus dieser ergibt sich die Leistungsfähigkeit eines Menschen, die Grundvoraussetzung für die Ausübung einer beruflichen Tätigkeit ist. In der ersten Etage wohnt die berufliche Kompetenz. Sie ergibt sich aus der Ausbildung einer Person, seiner Berufsbiographie und seinen beruflichen Kenntnissen. Sie kann darauf hinweisen, wie es um die Anpassungsfähigkeit an sich ändernde berufliche Anforderungen bestellt ist. Das zweite Stockwerk beheimatet die persönlichen Wertvorstellungen und Haltungen einer Person. Dies betrifft insbesondere ihre Vorstellungen von Respekt, Wertschätzung und Gerechtigkeit. Darüber, im dritten Stock, sind die Einstellungen und Haltungen gegenüber der Arbeitsstelle domiziliert. Wichtige Bestandteile sind die Arbeitsumgebung, der Arbeitsinhalt, die physische und psychische Arbeitsbelastung, die Arbeitsorganisation und das Verhältnis zu Vorgesetzten und Kollegen. Diese Punkte lassen sich unter dem Begriff «Jobzufriedenheit» zusammenfassen. Hieraus lassen sich Rückschlüsse auf die Identifikation mit der beruflichen Tätigkeit ziehen und die Motivation, sich für die Weiterführung der beruflichen Tätigkeit einzusetzen. Die Umgebung des «Hauses der Arbeitsfähigkeit» oder dessen Garten wird durch die familiären Verhältnisse einer Person, deren persönliches Umfeld und die Gesellschaft an sich gebildet. Störungen in einem Stockwerk des Hauses haben immer Auswirkungen auf die Stabilität des ganzen Gebäudes.

Mittels einer sorgfältigen und umfassenden Anamnese lässt sich das «Haus der Arbeitsfähigkeit», inklusive Garten erstellen. Zuerst beschreibt der Patient seine Gesundheitsbeschwerden frei und aus seiner Sicht. Er äussert sich zum bisherigen Verlauf und zur Prognose. Aus diesen Schilderungen lassen sich für einen Untersucher auch Rückschlüsse darauf ziehen, wie der Patient seine eigenen gesundheitlichen Ressourcen beurteilt und ob er selbst in Zukunft die Rückkehr in die bisherige Tätigkeit, aufgrund der gesundheitlichen Probleme, für möglich erachtet oder nicht.

Die persönliche Anamnese gibt Auskunft darüber, ob und wie ein Mensch mit Gesundheitsstörungen in der Vergangenheit umgegangen ist und welche allfälligen Konsequenzen aus diesen für die Erwerbstätigkeit entstanden sind, beispielsweise indem eine Umschulung erfolgte.

Die Berufsanamnese zeigt auf, ob die obligatorische Schulzeit regulär absolviert werden konnte oder irgendwelche Abweichungen zu verzeichnen sind. Es lohnt sich, den beruflichen Werdegang zu erfassen und dabei auch nicht beendete Ausbildungen, sowie Stellenwechsel oder berufliche Neuausrichtungen zu berücksichtigen. Das Datum des letzten Arbeitstages und ob das Arbeitsverhältnis gekündigt ist oder nicht, sind 
wichtige Angaben. All dies dient der Beurteilung der «Jobzufriedenheit». Ausserdem führt es zu Erkenntnissen, wie die betroffene Person subjektiv ihr aktuelles oder ehemaliges Arbeitsumfeld wahrnimmt.

Die Selbsteinschätzung der beruflichen und privaten Zukunftsperspektiven vermag Hinweise darauf zu liefern, ob sich ein Patient eine Wiedereingliederung in eine Erwerbstätigkeit und eine Weiterentwicklung in seinem Leben vorstellen kann oder ob er zu einer resignativen Haltung neigt. Im Falle einer Resignation stellt sich die Frage, wodurch diese bedingt ist. Das Vorliegen einer Depression kann mittels zweier Screeningfragen recht gut erhoben werden (siehe BMJ 2003; 327:144-6) ((Autor: mit diesen Angaben findet Pubmed nichts)).

Wichtig ist die Einbettung einer Person in ihr familiäres und gesellschaftliches Umfeld, sowie die finanzielle Situation. Ein schwieriger Beziehungsstatus, desolate Finanzen, rechtliche Auseinandersetzungen, Arbeitslosigkeit und Invalidität der Partnerin oder des Partners, eine nicht geklärte Kinderbetreuung oder Kinder, die nicht bei ihren Eltern leben dürfen, können erhebliche Reintegrationshindernisse ins Erwerbsleben darstellen. Diese Lebenserschwernisse gelten als invaliditätsfremde Faktoren. Sie begründen keine dauerhafte Arbeitsunfähigkeit.

Der Anamnese schliesst sich ein internistischer Allgemeinstatus an, ergänzt durch klinische Zusatzuntersuchungen, die sich nach den erhobenen Gesundheitsbeschwerden richten.

Die Ergebnisse der Exploration werden schliesslich in einem Bericht festgehalten. Dieser beinhaltet, nebst der gestellten Diagnosen, eine Beschreibung der Gesundheitssituation und die Beurteilung der Arbeitsfähigkeit, inklusive Begründung. Die Arbeitsfähigkeit wird einerseits in Prozent angegeben, andererseits in Stunden pro Tag. Hierdurch können vorhandene Leistungseinbussen transparent gemacht werden. Ein Beispiel ist die ganztägige Anwesenheit am Arbeitsplatz mit reduzierter Leistung. Der Beschäftigungsgrad einer Person wird nicht berücksichtigt, man geht generell von einer Anstellung in einem Vollpensum aus. Empfehlungen für Hilfsmittel, mit denen sich eine Arbeitsfähigkeit wieder herstellen oder erhalten lässt, sind in dem Bericht ebenfalls erwähnt. Schliesslich beinhaltet der Bericht Angaben zur medizinischen Weiterbetreuung, allenfalls zu einem medizinischen Rehabilitationsplan, zur Notwendigkeit weiterer Abklärungen und zur längerfristigen Prognose bezüglich Arbeitsfähigkeit. Ganz wichtig sind die Beschreibung vorhandener Ressourcen und Reintegrationshindernisse, auch wenn sich diese oft als Lebensumstände darstellen und somit invaliditätsfremd sind.

Hilfreich für die Beurteilung der Dauer einer Arbeitsunfähigkeit nach Unfällen ist der «Reintegrationsleitfaden Unfall» des Schweizerischen Versicherungsverbandes. Er beschreibt rund 500 Verletzungsmuster und gibt zu diesen prognostische Hinweise in Bezug auf den Normalverlauf, therapeutische Massnahmen, Komplikationsrisiken und eben die vermutliche Dauer einer Arbeitsunfähigkeit. Auch mögliche Langzeitfolgen und die Gefahr einer Invalidität durch das Erlittene sind beachtet. Die beschriebenen Normalverläufe vermögen jedoch nicht jeden Fall, mit all seinen potentiellen Abweichungen, darzustellen. 


\section{Fallbericht zur Zweitmeinung in der Beurteilung der Arbeitsfähigkeit}

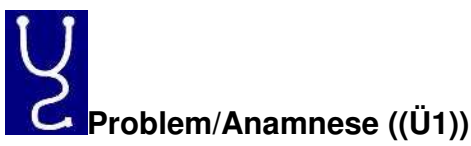

Eine 55-jährige Frau lebt seit 19 Jahren in der Schweiz. Sie ist Analphabetin und arbeitete nie ausser Haus. Der Ehemann ist nach einer Schulteroperation gesundheitlich eingeschränkt und wird von der Patientin betreut. Das Sozialamt kürzte die Ergänzungsleistungen mit dem Argument, die Hilfsbedürftigkeit des Ehemannes sei nicht derart ausgeprägt, dass seine Ehefrau den ganzen Tag zur Verfügung stehen müsse und sie sei in der Lage, sich um eine zumutbare Arbeit zu bemühen.

Die vom Hausarzt attestierte 50\%-Arbeitsfähigkeit erachtete die Familie der Patientin als unrealistisch hoch. Das Sozialamt beurteilte sie nicht rechtsgenüglich, da eine neutrale Expertise fehle. Deshalb erfolgt die Zuweisung durch den Hausarzt im Sinne einer Zweitmeinung zur «objektiven und neutralen» Beurteilung der Arbeitsfähigkeit. In seinem Schreiben erwähnt der Kollege die Diagnosen eines Diabetes mellitus, einer Adipositas, eines Status nach Nephrektomie mit normaler Leistung der verbleibenden Niere und eines Fibromyalgie-Syndroms.

\section{Anamnese ((Ü1))}

Die Patientin berichtet, dass sie chronische Schmerzen am ganzen Körper habe, die sie in ihrer Haushaltsarbeit häufig behinderten. Der Ehemann sei sehr hilfsbedürftig. Sie müsse inm helfen beim Rasieren, Anziehen und Baden, zudem müsse sie den ganzen Haushalt allein erledigen. Sie erzählt weiter, dass sie eigentlich gerne arbeiten gehen würde, auch damit sie aus dem Haus komme. Dies gehe jedoch nicht wegen der Hilfsbedürftigkeit des Ehemannes. Dieser sei zudem teilweise aggressiv und lehne eine auswärtige Hilfe wie die Spitex ab.

In der Systemanamnese beschreibt sie kein Fieber, keinen Gewichtsverlust, aber häufig auftretenden Nachtschweiss. Kopfschmerzen und Schwindel seien auch häufig zu beklagen. Gelegentlich komme. trockener Husten vor, aber kein Auswurf. Einen Thoraxdruck und Dyspnoe verspüre sie oft auch in Ruhe. Stuhlgang und Miktion seien unauffällig. Sie rauche nicht und trinke keinen Alkohol. Die Patientin ist verheiratet und Mutter von sechs Kindern.

\footnotetext{
Status ((Ü2))

Die 55-jährige Patientin ist in gutem $A Z$, hat einen $B M l$ von $34,2 \mathrm{~kg} / \mathrm{m}^{2}$, ist wach und allseits orientiert. Herz/Gefässe: Puls 68/min, regelmässig, BD 125/80 mmHg, Herztöne rein, keine Herzgeräusche, keine Strömungsgeräusche über den Carotiden, periphere Pulse allseits palpabel.

Lunge: sonorer Klopfschall bds, vesikuläres Atemgeräusch, keine Nebengeräusche.

Abdomen: weich, diffuse Druckdolenz im gesamten Abdomen ohne Abwehrspannung, keine Resistenzen, keine Organomegalien. Nierenlogen indolent. Rektal nicht untersucht.

Neurologie: Hirnnerven grobkursorisch unauffällig, Pupillen isokor mit prompter Lichtreaktion, Augenfolgebewegungen unauffällig, kein Meningismus, Armvorhalteversuch und Finger-Nase-Versuch unauffällig, Motorik, Kraft und Sensibilität an oberen und unteren Extremitäten unauffällig, Muskeleigenreflexe (BSR, PSR) seitengleich auslösbar. Druckdolenz an 14 von 18 Tenderpoints.
} 
Aktuelle Therapie ((Ü2))

Metformin Filmtabl. 850 mg 1-0-1, Gliclazid Tabl. 30 mg 1-0-0, Ramipril Tabl. 5 mg1-0-0, Esomeprazol 40 mg 0-0-

$1 / 4$, Ibuprofen 400 mg bei Bedarf, Paracetamol Tabl. 500 mg bei Bedarf, Butylscopolamin Drag 10 mg bei Bedarf.

Diagnosen/Beurteilung ((Ü1))

1. Weichteilrheumatische Beschwerden im Sinne eines Fibromyalgiesyndroms

2. Diabetes mellitus Typ II (ED: 2003)

3. Arterielle Hypertonie mit hypertensiver Herzkrankheit

- Echo 11/2008: linksventrikuläre Hypertrophie, diastolische Relaxationsstörung

- St.n. hypertensiver Entgleisung 11/2008

4. Hydronephrotische Schrumpfniere links

- St.n. Nephrektomie 2003

5. Thalassaemia minor (ED: 2005)

6. Refluxösophagits

Endorganschäden von Seiten des Diabetes mellitus lassen sich nicht erheben. Eine hypertensive Herzkrankheit verunmöglicht die Verrichtung von Hausarbeiten nicht. Weichteilrheumatische Beschwerden, resp. die Diagnose einer Fibromyalgie gelten nach aktueller bundesgerichtlicher Rechtsprechung als Beschwerden, die sich mit entsprechender Willensanstrengung überwinden lassen und keine dauerhafte Arbeitsunfähigkeit, resp. Leistungen der Invalidenversicherung rechtfertigen.

In der Tat meinen wir, dass durch ein angepasstes körperliches Training, das initial durch eine Fachperson in Physiotherapie instruiert und anschliessend eigenverantwortlich weitergeführt wird, eine Besserung der weichteilrheumatischen Beschwerden $\mathrm{zu}$ erreichen ist. Problematisch dabei ist die zu erwartende Schmerzexazerbation zu Beginn der medizinischen Massnahme. Wichtig wäre es, die Patientin gerade in dieser Phase zu stützen und zu motivieren. Ein enger Informationsaustausch mit den Therapiepersonen scheint sinnvoll. Ein weiteres Problem ist die Finanzierung eigenverantwortlicher Massnahmen, wie beispielsweise der Besuch eines Fitnessstudios.

Die Patientin nimmt bis heute keine ausschliesslich passive Rolle ein, sondern ist gemäss ihren Angaben, einer Doppelbelastung ausgesetzt durch die Verrichtung des Haushalts und die Betreuung des Ehemannes. Psychosoziale Belastungsfaktoren gelten als invaliditätsfremd und vermögen keine Leistungen der Invalidenversicherung zu begründen.

Zusammenfassend können wir keine langdauernde Arbeitsunfähigkeit aus gesundheitlichen Gründen attestieren, allenfalls vermögen Beschwerdeexazerbationen eine solche von jeweils wenigen Tagen Dauer zu begründen.

In diesem Fall geht es vordergründig um die Gesundheit und die Arbeitsfähigkeit der Patientin. Da sie bislang keiner Erwerbstätigkeit nachgeht, spielen die anderen Stockwerke des „Hauses der Arbeitsfähigkeit“ eine untergeordnete Rolle. Einen wichtigen Punkt stellt aber dessen Umgebung in Form der Pflegebedürttigkeit des Ehemannes, eines engen finanziellen Spielraums und einer wahrscheinlich nicht ganz einfachen Beziehung der Eheleute dar. Der Ehemann lehnt eine auswärtige Hilfe und Betreuung ab, uns sind Details nicht bekannt. Wir haben dem Hausarzt empfohlen, dessen tatsächliche Hilfsbedürftigkeit zu bestätigen und hiermit die Notwendigkeit der Anwesenheit der Patientin im häuslichen Umfeld dem Sozialamt gegenüber zu begründen. 


\section{Fragen zur Zweitmeinung in der Beurteilung der Arbeitsfähigkeit}

\section{Frage 1}

Welche Aussage über langdauernde Arbeitsunfähigkeit ist falsch? (Einfachauswahl, 1 richtige Antwort)
a) Sie greift das psychische und physische Wohlbefinden an.
b) Sie führt zu finanziellen Problemen durch fehlendes Einkommen.
c) Sie erhöht die Gefahr einer Suizidalität.
d) Sie verschlechtert generell die Lebensqualität.
e) Sie führt zu zwischenmenschlichen Problemen.

\section{Frage 2}

Welche Aussage zur Krankschreibung ist richtig? (Einfachauswahl, 1 richtige Antwort)

a) Das Thema «Krankschreibungen» ist für die meisten Ärzte leicht zu handhaben.

b) Die verschiedenen Rollen eines neutralen Experten und der behandelnden Person sind für Mediziner leicht zu bewältigen.

c) Die Krankheitskonzepte zwischen Patienten und Medizinern stimmen grundsätzlich überein.

d) Bei der Einschätzung der Dauer und des Ausmasses einer Arbeitsunfähigkeit sind die betreuenden Ärzte meist auf sich alleine gestellt.

e) Mediziner sind stets bestens über die berufliche Tätigkeit ihrer Patienten informiert.

\section{Frage 3}

Das «Haus der Arbeitsfähigkeit» besteht aus vier Stockwerken. Welches gehört nicht dazu? (Einfachauswahl, 1 richtige Antwort)
a) Gesundheit
b) Kompetenz
c) Werte
d) Arbeit
e) Gesellschaft

\section{Frage 4}

Welches gilt nicht als Reintegrationshindernis? (Einfachauswahl, 1 richtige Antwort)
a) Jahrelange Berufserfahrung
b) Arbeitslosigkeit des Partners
c) Desolate Finanzlage
d) Problematische Beziehung
e) Juristische Auseinandersetzungen

\section{Frage 5}

Über welchen Punkt muss der Bericht einer Zweitmeinung zur Beurteilung der Arbeitsfähigkeit keine Auskunft geben? (Einfachauswahl, 1 richtige Antwort)
a) Arbeitsfähigkeit in Prozent
b) Arbeitsfähigkeit in Stunden pro Tag 
c) Beschäftigungsgrad

d) Notwendige Hilfsmittel

e) Prognose bezüglich Arbeitsfähigkeit

\section{Korrespondenzadresse}

Dr. med. Hans-Joachim Thöne

Klinik für Innere Medizin

Universitätsspital

Rämistrasse 100

8091 Zürich

hans-joachim.thoene@usz.ch

\section{Bibliographie}

\section{((Autor: Referenzen bitte im Text mit eckigen Klammern [...] angeben))}

1. Bollag U, Rajeswaran A, Ruffieux C, Burnand B. Sickness certification in primary care - the physician's role. Swiss Med Wkly 2007;137:341-346.

2. Klipstein A, Schweizer Versicherung, März 2012; 40-41.

3. Guggenheim M, Birchler $P$, Schweizerischer Versicherungsverband, Reintegrationsleitfaden Unfall

4. Hyman MH, Melhorn JM, Talmage JB, AMA Guides to the Evaluation of Work Ability and Return to Work, ISBN-10:1-60359-530-9.

5. IImarinen J, Tuomi K: Past, present and future of work ability. In: Ilmarinen J, Lehtinen S (eds). Past, present and future of work ability. People and Work, Research Reports no. 65. Edited by Finnish Institute of Occupational Health, Helsinki, 2004, 1-25. 
DOI 10.1024/1661-8157/a001104

((PX 21_Rubrik CME-Antworten))

\section{Antworten zur Zweitmeinung in der Beurteilung der Arbeitsfähigkeit aus PRAXIS Nr. 20}

\section{Frage 1}

\section{Antwort b) ist richtig.}

Eine langdauernde Arbeitsunfähigkeit führt zu finanziellen Problemen, aber nicht wegen des fehlenden Einkommens. Dieses ist in den entwickelten Ländern durch Netzwerke von sozialen Institutionen sichergestellt. Das Übel sind Frustkäufe, die viele Menschen ohne Sinn und Aufgabe im Alltag tätigen. Aus ihnen entstehen die besagten Probleme mit Schulden durch Konsumkredite.

\section{Frage 2}

\section{Antwort d) ist richtig.}

Die Antworten auf die Frage 2 ergeben sich aus den Untersuchungen von Bollag $U$ et al. [1]. Kernaussagen des Artikels sind, dass sind Krankschreibungen für Mediziner generell keine einfache Tätigkeit sind. Bei der Einschätzung der Dauer und des Ausmasses einer Arbeitsunfähigkeit sind die betreuenden Ärzte meist auf sich alleine gestellt. Es kommt vor, dass sie in einen Konflikt geraten zwischen ihren Rollen als Behandelnde und Fürsorgende ihrer Patienten einerseits und neutralen Experten, die auch rechtliche Vorgaben mit einzubeziehen haben, sowie die Interessen der Allgemeinheit, andererseits. Zudem bestehen ärztlicherseits häufig keine Kenntnisse bezüglich des genauen Tätigkeitsprofils, das Patienten im beruflichen Alltag zu erfüllen haben. Hinderlich sind subjektive Krankheitskonzepte einzelner Patienten, die teilweise von der Lehrmeinung erheblich abweichen können. Zu guter Letzt wird das Thema einer Arbeitsunfähigkeit oftmals am Schluss einer Konsultation kurz vor oder auch nach der Verabschiedung thematisiert, so dass ein erheblicher Zeitdruck besteht und wenig Möglichkeit vorhanden sind, sich gedanklich mit der Thematik auseinanderzusetzen.

\section{Frage 3}

\section{Antwort e) ist richtig.}

Die Antworten a-d stellen die vier Stockwerke des «Hauses der Arbeitsfähigkeit» dar. Die Umgebung des «Hauses der Arbeitsfähigkeit» oder dessen Garten wird durch die familiären Verhältnisse einer Person, deren persönliches Umfeld und die Gesellschaft an sich gebildet.

\section{Frage 4}

Antwort a) ist richtig.

Ein schwieriger Beziehungsstatus, desolate Finanzen, rechtliche Auseinandersetzungen, Arbeitslosigkeit und Invalidität der Partnerin oder des Partners, eine nicht geklärte Kinderbetreuung oder Kinder, die nicht bei ihren Eltern leben dürfen, können erhebliche Reintegrationshindernisse ins Erwerbsleben darstellen. Demgegenüber sind eine abgeschlossen Ausbildung, eine langjährige Berufserfahrung, eine hohe Identifikation mit der Arbeit, etc. als Ressourcen zu werten.

\section{Frage 5}

Antwort c) ist richtig. 
Bei der Beurteilung der Arbeitsfähigkeit einer Person wird der Beschäftigungsgrad nicht berücksichtigt, man geht generell von einer Anstellung in einem Vollpensum aus. Zu den anderen Punkten sollte Stellung genommen werden.

1. Bollag U, Rajeswaran A, Ruffieux C, Burnand B: Sickness certification in primary care - the physician's role. Swiss Med Wkly 2007; 137: 341-346. 